\section{Development and validation of a modified Frailty Risk Index as a predictor of mortality in rural elderly people}

\author{
Sunil Kumar ${ }^{1}$, MD, PhD, Shraddha Jain ${ }^{2}, \mathrm{MS}, \mathrm{PhD}$, Anil Wanjari ${ }^{1}, \mathrm{MD}$,
} Syamsunder Mandal ${ }^{3}$, $\mathrm{PhD}$

\begin{abstract}
Background. Frailty is a reversible age-related condition characterised by declines across multiple physiologic systems and associated with an increased risk of mortality or unplanned hospitalisation. We developed and validated a new frailty index that is easy to apply in elderly people in rural India and has predictive accuracy for all-cause mortality at 6 and 12 months.
\end{abstract}

Methods. 1000 participants aged $>60$ years who were admitted to a geriatric unit of a rural hospital for screening or treatment of any illness were recruited for development and validation of the Frailty Index in Rural Elderly - Mental status, Activities of daily living, Depression, and Events (FIRE-MADE), which combines the Cumulative Deficits Model and the Comprehensive Geriatric Assessment Model and takes into account of four domains: physical, cognitive, psychosocial, and functional.

Results. The FIRE-MADE score increased with age and was higher in men than in women. Higher FIRE-MADE score was associated with higher rates of mortality and unplanned hospitalisation. In multivariable analysis, predictors for mortality were Mini-Mental State Examination, activities of daily living, ischaemic heart disease, history of stroke, and polypharmacy. In Kaplan-Meier survival analysis, 4.9\%, 7.1\%, and $16.7 \%$ of participants with mild (FIRE-MADE score, $0.3-0.4$ ), moderate $(0.5-0.6)$, and severe (>0.7) frailty died at the end of 1 year. The cut-off values for mortality and unplanned hospitalisation were 0.32 and 0.27 , respectively, with the area under the receiver operating characteristic curve for the severity being 0.883 and 0.794, respectively. Compared with the Longitudinal Aging Study Amsterdam frailty index score in predicting mortality and unplanned hospitalisation, the FIRE-MADE score had positive predictive values of $88.89 \%$ and $88.89 \%$, respectively, and negative predictive values of $65.93 \%$ and $41.10 \%$, respectively. Association between the two indices was strong.

Conclusions. FIRE-MADE is easy to apply in clinical practice as a screening tool to detect frailty in elderly people in rural India.

Key words: Frail elderly; Hospitalization; Mortality

\section{ORIGINAL ARTICLE}

\footnotetext{
Department of Medicine, Jawahar Lal Nehru Medical College, Datta Meghe Institute of Medical Science, Wardha, Maharashtra, India

2 Department of Otorhinolaryngology, Jawahar Lal Nehru Medical College, Datta Meghe Institute of Medical Science, Wardha, Maharashtra, India

Department of Biostatistics, Chittaranjan National Cancer Institute, Kolkata, West Bengal, India
}

Correspondence to: Prof Sunil Kumar, Department of Medicine, Jawahar Lal Nehru Medical College, Datta Meghe Institute of Medical Science (deemed to be a university), Wardha, Maharashtra, India. Email: sunilkumarmed@gmail.com 


\section{INTRODUCTION}

Frailty is a reversible age-related condition characterised by declines across multiple physiologic systems and associated with an increased risk of mortality or unplanned hospitalisation. The term 'frail' is intended to identify vulnerable elderly people at high risk of falls, disability, hospitalisation, and mortality. Nonetheless, frailty is not synonymous with age or disease. It is a geriatric syndrome and involves excess healthcare costs from consultations, polypharmacy, and hospitalisation. ${ }^{1}$ Frailty confers loss of independence in activities of daily living and death when exposed to stress. Nonetheless, physical frailty is potentially reversible..$^{1-3}$

Numerous definitions and assessment tools for frailty have been developed. The Fried Frailty Phenotype Framework has been commonly used and extensively validated, ${ }^{2}$ but it is considered not readily applicable in primary care practice. ${ }^{4,5}$ It measures only five variables: weight loss, exhaustion, grip strength, walking speed, and physical activity. ${ }^{2}$ The presence of any three variables is defined as frailty $(0=$ non-frail, 1-2=pre-frail, and 3-5=frail). ${ }^{2}$ Dichotomisation on continuous scales (grip strength, walking speed, and physical activity) is done retrospectively according to the lowest 12th percentile rule, with further stratifications. This requires expertise in statistics and a reference sample, which is not easy for primary care practitioners. ${ }^{6}$
Owing to the ageing population and rising healthcare costs, health promotion and disability prevention is emphasised. ${ }^{6,7}$ To provide early intervention and/or multidisciplinary management at the primary care level, it is important to identify at-risk elderly people based on frailty, which fits well with the physical and psychosocial model. ${ }^{8}$ However, appropriate frailty metrics for primary care are lacking, and general practitioners are in need of easy instruments for frailty. ${ }^{9}$

The Cumulative Deficit Model identifies frailty based on variables (such as symptoms, signs, diseases, disabilities, and abnormal laboratory values) collectively referred to as deficits. ${ }^{10}$ The original model has 92 variables, but this can be reduced to 30 without loss of predictive validity. ${ }^{11,12}$ A frailty index is calculated based on the presence or absence of each variable as a proportion of the total. The criteria for variable inclusion in the frailty index are: (1) biologically plausible; (2) accumulates with age; and (3) does not saturate too early (ie, the prevalence of the deficit does not reach $100 \%$ before older age). ${ }^{13-15}$

In India, there are $>100$ million elderly people who often have comorbidities that are often neglected; $10 \%$ of them are admitted to hospital each year. ${ }^{16,17}$ This study aimed to develop and validate the Frailty Index in Rural Elderly - Mental status, Activities of daily living, Depression, and Events (FIRE-MADE)

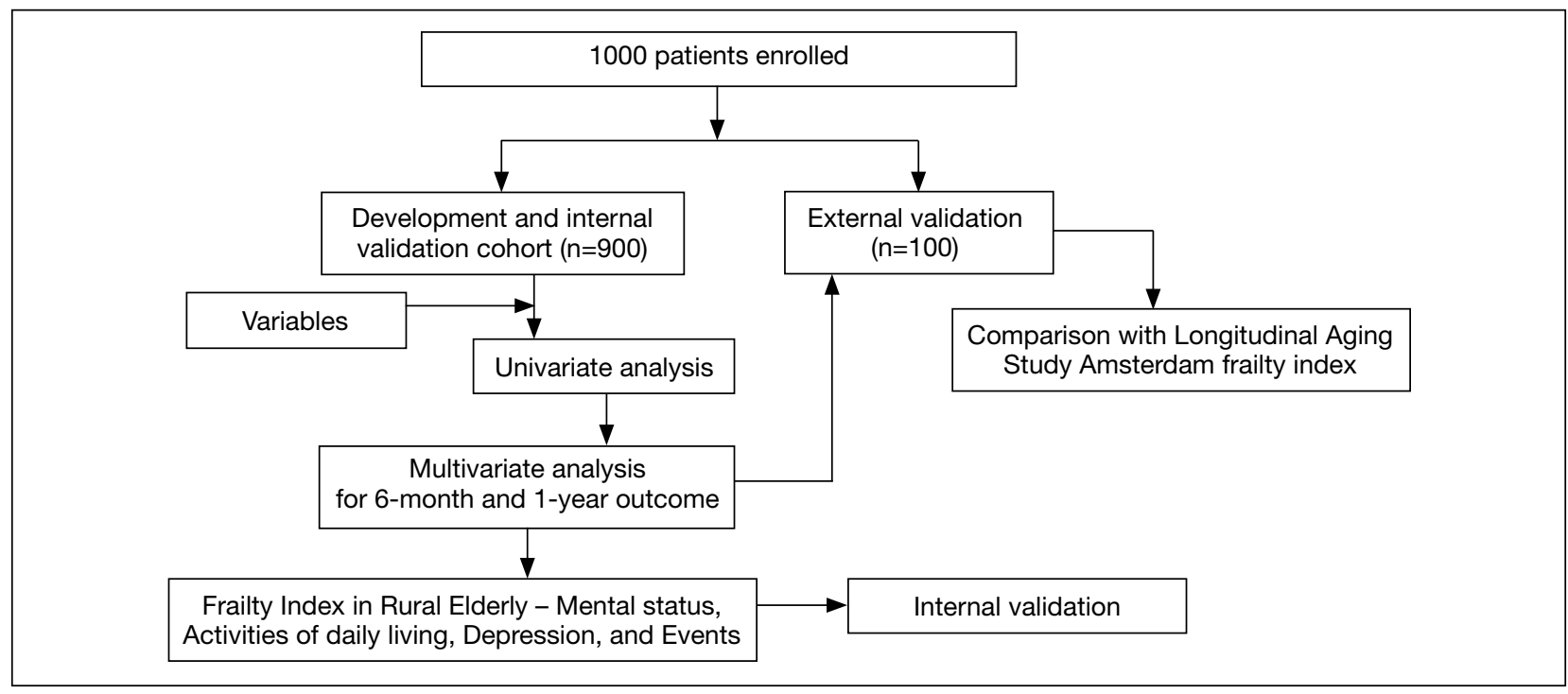

Figure 1. Flowchart of the study 
to identify predictors of frailty to predict all-cause mortality and unplanned hospitalisation in rural elderly people, with an aim of health promotion and disability prevention.

\section{MATERIALS AND METHODS}

This study was approved by the ethics committee of the Jawahar Lal Nehru Medical College (DMIMS (DU)/IEC/2014-15/859). Written informed consent was obtained from each participant. This longitudinal observational study was carried out from January 2015 to December 2017, with participants followed up at 6 and 12 months. 1000 elderly people aged $>60$ years who were admitted to our hospital for screening and treatment of any illness were recruited and randomly allocated to either the development cohort $(n=900)$ or the validation cohort $(n=100)$. Both cohorts had same study protocol (FIGURE 1). The sample size was calculated based on the study by Hoogendijk et $\mathrm{al}^{18}$ in which the prevalence of frailty was $23.9 \%$. For $p=0.239$, the number of subjects required was 900 with a power of $88 \%$.

\section{Development of FIRE-MADE}

The Cumulative Deficits Model and the Comprehensive Geriatric Assessment Model were incorporated to form four domains: physical, cognitive, psychosocial, and functional. ${ }^{19,20}$ The physical domain incorporated health-related deficits (stroke, diabetes, heart attack) were taken from the Cumulative Deficits Model, whereas the cognitive, psychosocial (emotional), and functional (activities of daily living) domains were taken from the Comprehensive Geriatric Assessment Model. Based on literature review and clinical relevance, we identified 10 parameters for development of FIREMADE: (1) mental status, (2) activities of daily living, (3) depression, and events like (4) polypharmacy, (5) diabetes mellitus, (6) ischaemic heart disease, (7) chronic obstructive pulmonary disease / asthma, (8) stroke, (9) cancer, (10) others. Presence or absence of each parameter was indicated by 1 or 0 [TABLE $\mathbf{I}$ ].

For internal (content) validation, feedbacks were taken from experts. Each item was tested for its association with mortality using univariate analysis.

TABLE 1

Parameters of Frailty Index in Rural Elderly - Mental status, Activities of daily living, Depression, and Events

\begin{tabular}{ll}
\hline Parameter & Score \\
\hline $\begin{array}{l}\text { Mini-Mental State Examination score of }<27 \text { (impaired) } \\
\text { Help needed with activities of daily living score }\end{array}$ & $\mathrm{No}=0$, Yes $=1$ \\
Feeding & $\mathrm{No}=0$, Yes $=1$ \\
Dressing & $\mathrm{No}=0$, Yes $=1$ \\
Bathing & $\mathrm{No}=0$, Yes $=1$ \\
Going to toilet & $\mathrm{No}=0$, Yes $=1$ \\
Urinary continence & $\mathrm{No}=0$, Yes $=1$ \\
Transferring & $\mathrm{No}=0$, Yes $=1$ \\
Geriatric Depression Scale score of $>5$ & $\mathrm{No}=0$, Yes $=1$ \\
Events & \\
Polypharmacy & $\mathrm{No}=0$, Yes $=1$ \\
Diabetes mellitus & $\mathrm{No}=0$, Yes $=1$ \\
Ischaemic heart disease & $\mathrm{No}=0$, Yes $=1$ \\
Chronic obstructive pulmonary disease / asthma & $\mathrm{No}=0$, Yes $=1$ \\
Stroke & $\mathrm{No}=0$, Yes $=1$ \\
Cancer & $\mathrm{No}=0$, Yes $=1$ \\
Others & $\mathrm{No}=0$, Yes $=1$ \\
\hline
\end{tabular}


Multivariate logistic regression analysis was used to determine predictors of mortality after adjusting for confounders such as age, sex, education, and blood pressure. The predictors of mortality were then included in FIRE-MADE.

The FIRE-MADE score was calculated for each participant by summing the number of deficit parameters and dividing the total number of parameters $(n=10)$. Based on the 32-item Longitudinal Aging Study Amsterdam (LASA) instrument, ${ }^{21}$ the cut-off value of 0.25 was used and four groups were devised: fit $(<0.25)$, mild frailty (0.3-0.4), moderate frailty (0.5-0.6), and severe frailty $(>0.7)$. The four groups were tested for the predictive validity in terms of mortality and unplanned hospitalisation. Hazard ratios (HR) for mortality and unplanned hospitalisation at 6 and 12 months were estimated. Kaplan-Meier survival analysis was performed using the log-rank test to compare the survival pattern of different frailty groups. Receiver operating characteristic (ROC) curve was used to determine the cut-off value of the FIRE-MADE score for mortality and unplanned hospitalisation. These cut-off values were then validated in the validation cohort.

\section{Validation of FIRE-MADE}

For testing the predictive validity of FIRE-MADE, Kaplan-Meier curves for mortality and unplanned hospitalisation were plotted for the two risk groups (fit and frail) of the validation cohort, based on the cut-off values devised from the development cohort.

In addition, the validation cohort was retrospectively assessed (based on records) with the LASA frailty index, ${ }^{21}$ which has four functional domains: physical, cognitive, emotional, and social functioning. The LASA frailty index has been validated for use as external reference as a predictor of mortality. Prognostic score calculation and classification of the risk groups (fit and frail) were repeated in the validation cohort using ROC curves. FIRE-MADE was compared with the LASA frailty index to determine the sensitivity, specificity, positive and negative predictive values, and diagnostic accuracy.

\section{Parameters of FIRE-MADE}

Data collected included socio-demographics (age, sex, education), the presence of diabetes, chronic obstructive pulmonary disease/asthma, stroke, cancers, polypharmacy, cardiac diseases (ischaemic heart disease), other conditions (arthritis, fall, incontinence), and medications used in the past year. Polypharmacy was defined as the use of $\geq 5$ medications with different anatomic therapeutic chemical classifications over the past 6 months. ${ }^{16}$ Depressive symptoms was measured using the Geriatric Depression Scale short version, ${ }^{22,23}$ with a score of $>5$ indicating a clinically significant level of depressive symptoms. Cognitive function was evaluated using the Hindi adaptation of the MiniMental State Examination (MMSE) ${ }_{1}{ }^{24}$ with a score of $<27$ indicating cognitive impairment. Functional dependency was assessed using self-reported difficulty and requiring help on basic activities of daily living. ${ }^{25}$ Hospitalisation was based on patients' self-report and confirmed by hospital discharge ticket or prescription tickets. Mortality was confirmed by telephonic contact with relatives or registers of the municipalities where respondents were living.

\section{Statistical analysis}

Statistical analysis was performed using Epi Info 7.2.2.2. Chi-squared test was used to determine association of different parameters. Z-test was used for comparison of proportions, and t-test was used

TABLE 2

Scores of Frailty Index in Rural Elderly - Mental status, Activities of daily living, Depression, and Events

\begin{tabular}{lcccc}
\hline Age group, $y$ & Overall $(n=900)$ & Men $(n=468)$ & Women $(n=432)$ & $p$ Value \\
\hline $60-65$ & $0.30 \pm 0.15(n=423)$ & $0.30 \pm 0.18(n=162)$ & $0.31 \pm 0.13(n=261)$ & 0.45 \\
$66-70$ & $0.35 \pm 0.17(n=297)$ & $0.34 \pm 0.15(n=162)$ & $0.37 \pm 0.20(n=135)$ & $<0.01$ \\
$71-75$ & $0.48 \pm 0.21(n=144)$ & $0.49 \pm 0.20(n=117)$ & $0.43 \pm 0.26(n=27)$ & $<0.001$ \\
$>75$ & $0.62 \pm 0.23(n=36)$ & $0.56 \pm 0.24(n=27)$ & $0.80 \pm 0.01(n=9)$ & $<0.001$ \\
Total & $0.36 \pm 0.19$ & $0.37 \pm 0.20$ & $0.35 \pm 0.18$ & 0.38 \\
One-way ANOVA (F value, p value) & $F_{3,896}=62.12, p<0.0001$ & $F_{3,464}=36.96, p<0.0001$ & $F_{3,428}=29.34, p<0.0001$ & \\
\hline
\end{tabular}


TABLE 3

Multivariate logistic regression for predictors of mortality

\begin{tabular}{lccc}
\hline Variables & Regression coefficient $(\beta)$ & $\mathrm{p}$ Value & Exp( $\beta$ [odds ratio $(95 \% \mathrm{Cl})$ ] \\
\hline Mini-Mental State Examination & 4.594 & 0.001 & $8.862(3.308-19.327)$ \\
Activities of daily living & 1.339 & 0.041 & $3.815(1.010-16.162)$ \\
Depression & 0.124 & 0.788 & $1.132(0.457-2.808)$ \\
Ischaemic heart disease & -2.179 & 0.001 & $4.020(1.041-3.309)$ \\
Chronic obstructive pulmonary disease & -0.782 & 0.071 & $0.457(0.195-1.071)$ \\
Diabetes & 0.637 & 0.226 & $1.892(0.674-5.308)$ \\
History of stroke & 2.055 & 0.003 & $7.807(2.001-30.469)$ \\
Polypharmacy & 3.689 & 0.001 & $39.994(12.622-126.726)$ \\
Cancer & -0.411 & 0.386 & $0.663(0.261-1.681)$ \\
Other comorbidities & -0.133 & 0.783 & $0.875(0.339-2.262)$ \\
Constant & -10.265 & 0.001 & 0.001 \\
\hline
\end{tabular}

for comparison of means. Multiple logistic regression analysis was used to determine predictors of frailty after adjusting for confounders, with odds ratio (OR) and $95 \%$ confidence interval (CI) calculated. Oneway analysis of variance (ANOVA) followed by Tukey test was used to compare more than two means at a time. ROC analysis was used to determine the cut-off values for mortality and unplanned hospitalisation. Kaplan-Meier survival analysis by log-rank test was used to compare the survival pattern of different risk groups. Sensitivity, specificity, positive and negative predictive values were calculated. A $p$ value of $<0.05$ was considered statistically significant.

\section{RESULTS}

Of the 1000 participants aged 60 to 85 (mean, 67.11; median, 66; standard deviation, 5.11) years, $47 \%$ were aged $60-65$ years, $33 \%$ were aged $66-70$ years, $16 \%$ were aged $71-75$ years, and $4 \%$ were aged $>75$ years $(\mathrm{p}<0.05)$.

In the development cohort, the FIRE-MADE score increased with age and was higher in men than in women (both $\mathrm{p}<0.0001$, one-way ANOVA, TABLE 2). The prevalence of FIRE-MADE score of $\geq 0.25$ was associated with age and was higher in men than in women (both $\mathrm{p}<0.0001$, Chi-squared test). Higher FIRE-MADE score was associated with higher rates of mortality and unplanned hospitalisation (both $\mathrm{p}<0.0001$, Chi-squared test).

In multivariable analysis, after adjusting for confounders, predictors for mortality were MMSE (OR=8.86, 95\% CI=3.30-19.32, $\mathrm{p}=0.0001)$, activities of daily living (OR=3.81, 95\% $\mathrm{CI}=1.01-16.16, \mathrm{p}=0.041)$, ischaemic heart disease $(\mathrm{OR}=4.02,95 \% \mathrm{CI}=1.04-3.31$, $\mathrm{p}=0.001)$, history of stroke $(\mathrm{OR}=7.80,95 \% \mathrm{CI}=2.00$ $30.46, \mathrm{p}=0.003)$, and polypharmacy (OR=39.99, 95\% $\mathrm{CI}=12.62-126.72, \mathrm{p}=0.001)$ [TABLE 3].

In Kaplan-Meier survival analysis, 4.9\%, 7.1\%, and $16.7 \%$ of participants with mild (FIRE-MADE score, $0.3-0.4)$, moderate (0.5-0.6), and severe $(>0.7)$ frailty died at the end of 1 year. The survival rate decreased with increased FIRE-MADE score (FIGURE 2). Different risk groups had different survival patterns (log-rank=218.54, $\mathrm{p}<0.000$ ). The cut-off

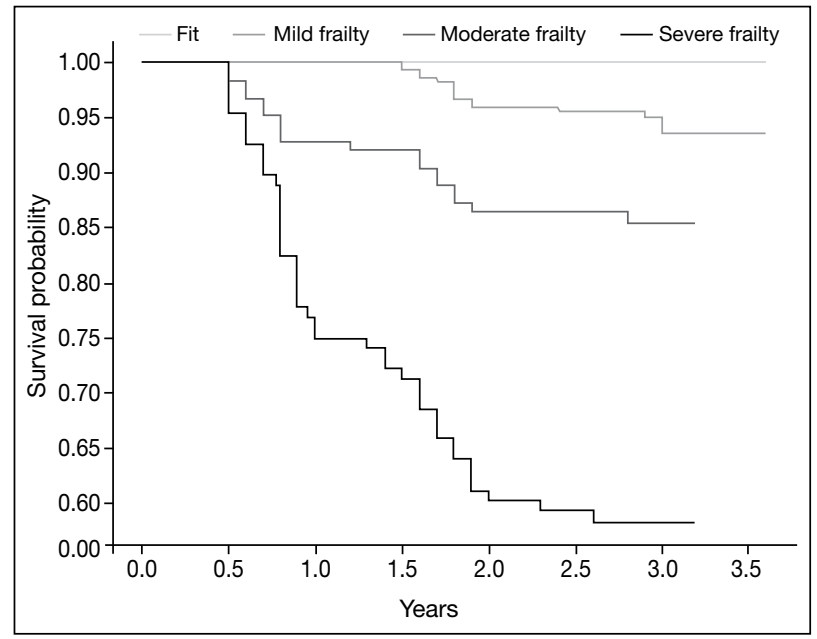

FIGURE 2. Kaplan-Meier survival curves for fit and mild, moderate, and severe frailty groups based on the Frailty Index in Rural Elderly - Mental status, Activities of daily living, Depression, and Events 
(a)

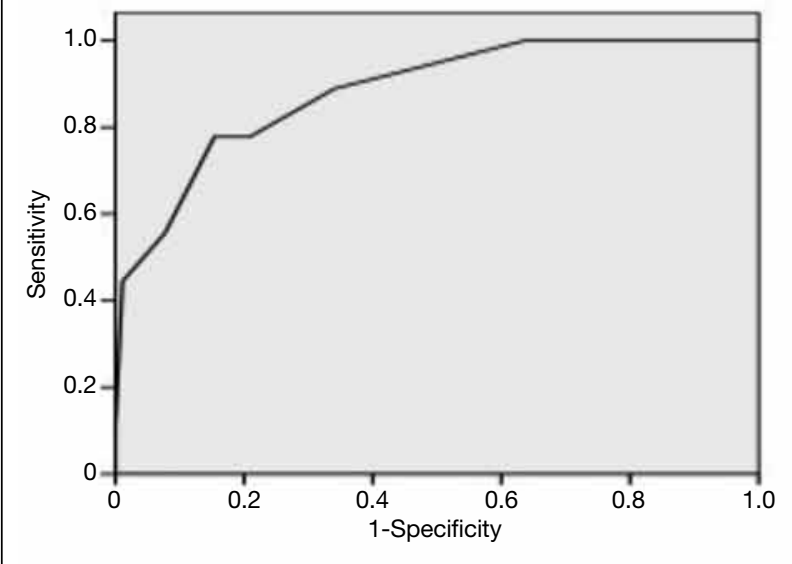

(b)

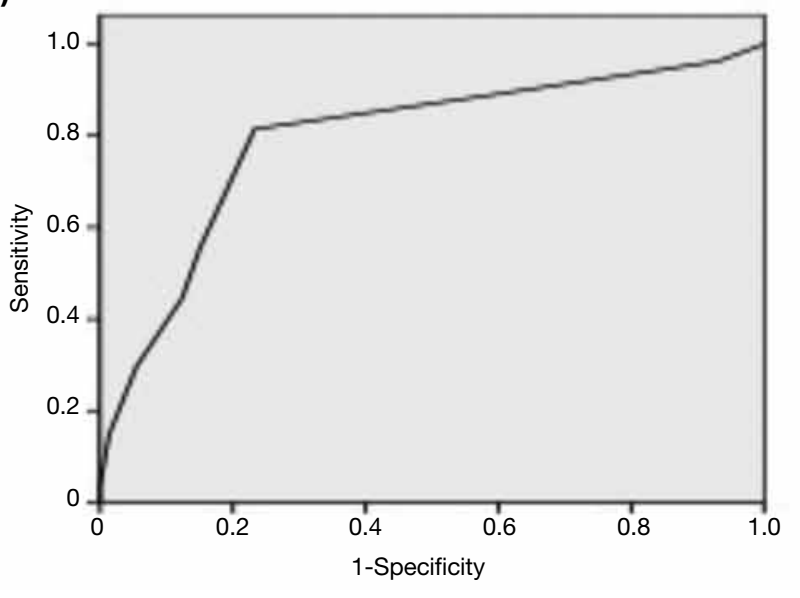

FiguRE 3. The cut-off values for (a) mortality and (b) unplanned hospitalisation were 0.32 and 0.27 , respectively, with the area under the receiver operating characteristic curve for the severity being 0.883 and 0.794 , respectively.

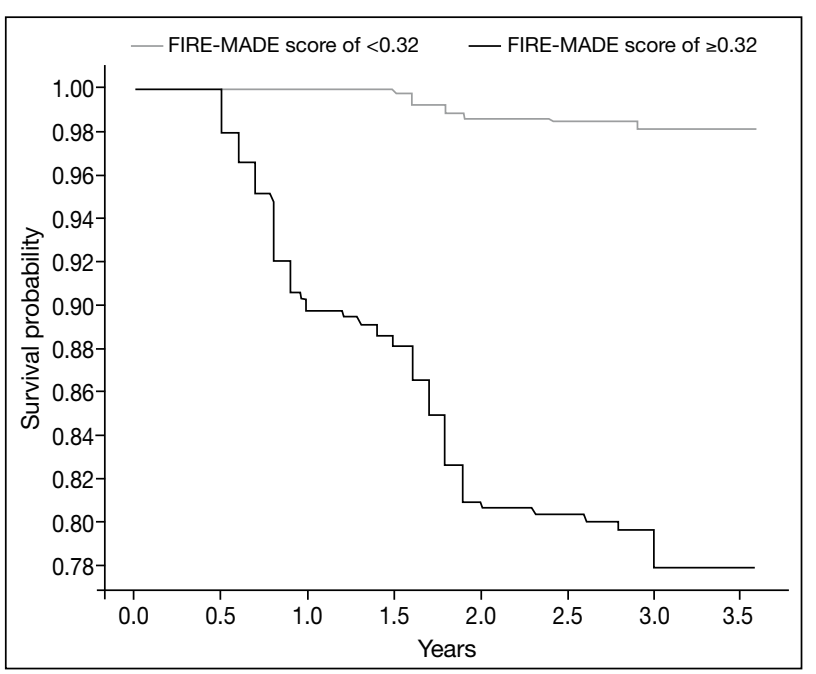

FIGURE 4. Kaplan-Meier survival curve for mortality with a cut-off value of 0.32

values for mortality and unplanned hospitalisation were 0.32 and 0.27 , respectively, and the areas under the ROC curve for the severity were 0.883 and 0.794 , respectively (FIGURE 3).

In the validation cohort, the 1-year mortality pattern differed significantly in those with FIREMADE score of $\geq 0.32$ or $<0.32$ (log rank=96.33, $\mathrm{p}<0.001$, Figure 4). The unadjusted and adjusted Cox proportional hazard ratio for the cut-off value of 0.32 was $13.90(95 \% \mathrm{CI}=6.95-27.80, \mathrm{p}<0.001)$ and 9.77 (95\% CI=4.82-19.80, $\mathrm{p}<0.001)$, respectively. The unplanned hospitalisation pattern differed significantly in those with FIRE-MADE score of $\geq 0.27$ or $<0.27$ ( $\log$ rank=8.52, $\mathrm{p}=0.003$ ). The unadjusted and adjusted Cox proportional hazard ratio for the cut-off value of 0.27 was $0.22(95 \% \mathrm{CI}=0.06-0.73$, $\mathrm{p}=0.013)$ and $0.21 \quad(95 \% \mathrm{CI}=0.06-0.70, \mathrm{p}=0.011)$, respectively.

Compared with the LASA frailty index score, the FIRE-MADE score had a positive predictive value of $88.89 \%$ and $88.89 \%$, and a negative predictive value of $65.93 \%$ and $41.10 \%$ in predicting mortality and unplanned hospitalisation, respectively (TABLE 4). Association between the two indices was strong.

\section{DISCUSSION}

FIRE-MADE with 10 health deficit items is easy to apply in rural India, taking into account of social, psychological, mental, treatment-related, and health deficits in affecting mortality and unplanned hospitalisation. Content validation included all the relevant items and excluded repeat items. Polypharmacy was not included in previous frailty indices such as the LASA frailty index, but we included polypharmacy in developing FIRE-MADE, because polypharmacy has been reported to be associated with frailty in elderly people. ${ }^{14,17,19-21,26,27}$ Construct validation of each item was performed using univariate and multivariate analyses, and predictors of frailty (MMSE, activities of daily living, ischaemic heart disease, history of stroke, and polypharmacy) 
TABLE 4

Comparison of external validity of Frailty Index in Rural Elderly - Mental status, Activities of daily living, Depression, and Events (FIRE-MADE) with the Longitudinal Aging Study Amsterdam (LASA) frailty index

\begin{tabular}{|c|c|c|c|c|c|c|c|}
\hline Outcome & $\begin{array}{l}\text { Area under } \\
\text { curve }\end{array}$ & $\begin{array}{l}\text { Cut-off } \\
\text { value }\end{array}$ & $\begin{array}{c}\text { Diagnostic } \\
\text { accuracy, } \\
\%\end{array}$ & $\begin{array}{c}\text { Sensitivity, } \\
\%\end{array}$ & $\begin{array}{c}\text { Specificity } \\
\%\end{array}$ & $\begin{array}{l}\text { Positive } \\
\text { predictive } \\
\text { value, \% }\end{array}$ & $\begin{array}{l}\text { Negative } \\
\text { predictive } \\
\text { value, \% }\end{array}$ \\
\hline \multicolumn{8}{|l|}{ FIRE-MADE } \\
\hline Mortality & 0.883 & 0.32 & 68 & 20.51 & 98.36 & 88.89 & 65.93 \\
\hline Unplanned hospitalisation & 0.794 & 0.27 & 54 & 35.32 & 90.91 & 88.89 & 41.10 \\
\hline \multicolumn{8}{|l|}{ LASA frailty index } \\
\hline Mortality & 0.948 & 0.34 & 69 & 21.05 & 98.39 & 88.89 & 67.03 \\
\hline Unplanned hospitalisation & 0.833 & 0.31 & 73 & 50.00 & 96.00 & 92.59 & 65.75 \\
\hline
\end{tabular}

were identified after adjusting for confounders. Although depression, chronic obstructive pulmonary disease, diabetes mellitus, cancer, and other comorbidities were not predictive of mortality, these items were kept in FIRE-MADE, as exclusion may affect the content validity. FIRE-MADE was used to classify frailty (fit, mild, moderate, and severe frailty) and then to predict mortality and unplanned hospitalisation at 6 and 12 months. ROC curves were constructed and areas under the ROC curve were calculated for the cut-off values of mortality (0.32) and unplanned hospitalisation (0.27). These cut-off values were validated in the validation cohort. This established the'predictive validity' component of the 'criterion validity' of FIRE-MADE. The initial arbitrary cut-off value of 0.25 was close to the externally validated cut-off values. Criterion validity was established by assessing both 'concurrent validity' and 'predictive validity'. The 'concurrent validity' was assessed by comparing FIRE-MADE with the LASA frailty index for sensitivity, specificity, and positive and negative predictive values. Association between the two indices was strong.

One limitation of our study is that participants were recruited from only one tertiary centre in rural India. In addition, the follow-up duration was too short to conclude the cut-off value for risk stratification.

\section{CONCLUSION}

FIRE-MADE is easy to apply in clinical practice as a screening tool to detect frailty in elderly people in rural India.

\section{DECLARATION}

The authors have no conflict of interest to disclose.

\section{REFERENCES}

1. Ahmed N, Mandel R, Fain MJ. Frailty: an emerging geriatric syndrome. Am J Med 2007;120:748-53. Crossref

2. Fried LP, Tangen CM, Walston J, et al. Frailty in older adults: evidence for a phenotype. J Gerontol A Biol Sci Med Sci 2001;56:M146-56. Crossref

3. Hubbard RE, O'Mahony MS, Woodhouse KW. Characterising frailty in the clinical setting: a comparison of different approaches. Age Ageing 2009;38:115-9. Crossref

4. Abellan van Kan G, Rolland YM, Morley JE, Vellas B. Frailty: toward a clinical definition. J Am Med Dir Assoc 2008;9:712. crossref

5. Rockwood K, Mitnitski A. Frailty defined by deficit accumulation and geriatric medicine defined by frailty. Clin Geriatr Med 2011;27:17-26. Crossref

6. Bergman H, Ferrucci L, Guralnik J, et al. Frailty: an emerging research and clinical paradigm: issues and controversies. J Gerontol A Biol Sci Med Sci 2007;62:731-7. Crossref

7. Chin A Paw MJ, Dekker JM, Feskens EJ, Schouten EG, Kromhout D. How to select a frail elderly population? A comparison of three working definitions. J Clin Epidemiol 1999;52:1015-21. Crossref

8. Turner G, Clegg A; British Geriatrics Society; Age UK; Royal College of General Practioners. Best practice guidelines for the management of frailty: a British Geriatrics Society, Age UK and Royal College of General Practitioners report. Age Ageing 2014;43:744-7. Crossref

9. Fillit $\mathrm{H}$, Butler RN. The frailty identity crisis. J Am Geriatr Soc 2009;57:348-52. Crossref

10. Clegg A, Bates C, Young J, et al. Development and validation of an electronic frailty index using routine primary care electronic health record data. Age Ageing 2016;45:353-60. Crossref

11. Mitnitski AB, Mogilner AJ, Rockwood K. Accumulation of deficits as a proxy measure of aging. ScientificWorldJournal 2001;1:32336. Crossref

12. Song X, Mitnitski A, Rockwood K. Prevalence and 10year outcomes of frailty in older adults in relation to deficit accumulation. J Am Geriatr Soc 2010;58:681-7. Crossref

13. Searle SD, Mitnitski A, Gahbauer EA, Gill TM, Rockwood K. A standard procedure for creating a frailty index. BMC Geriatr 2008;8:24. Crossref 
14. Rockwood K, Song X, MacKnight C, et al. A global clinical measure of fitness and frailty in elderly people. CMAJ 2005; 173:489-95. Crossref

15. Cesari M, Gambassi G, van Kan GA,Vellas B.The frailty phenotype and the frailty index: different instruments for different purposes. Age Ageing 2014;43:10-2. Crossref

16. India's Aging Population. Available from: http://www.prb. org/Publications/Reports/2012/india-older-population.aspx. Accessed July 2018.

17. KashikarY, Nagarkar A. Prevalence and determinants of frailty in older adults in India. Indian J Gerontol 2016;30:364-81.

18. Hoogendijk EO, van der Horst HE, Deeg DJ, et al. The identification of frail older adults in primary care: comparing the accuracy of five simple instruments. Age Ageing 2013;42:2625. Crossref

19. Kulminski AM, Ukraintseva SV, Kulminskaya IV, Arbeev KG, Land K, Yashin AI. Cumulative deficits better characterize susceptibility to death in elderly people than phenotypic frailty: lessons from the Cardiovascular Health Study. J Am Geriatr Soc 2008;56:898-903. Crossref

20. Rockwood K, Mitnitski A. Frailty in relation to the accumulation of deficits. J Gerontol A Biol Sci Med Sci 2007;62:722-7. Crossref

21. Hoogendijk EO, Theou O, Rockwood K, Onwuteaka-Philipsen
BD, Deeg DJH, Huisman M. Development and validation of a frailty index in the Longitudinal Aging Study Amsterdam. Aging Clin Exp Res 2017;29:927-33. Crossref

22. Joshi K, Kumar R, Avasthi A. Morbidity profile and its relationship with disability and psychological distress among elderly people in Northern India. Int J Epidemiol 2003;32:978-87. Crossref

23. Kamble SV, Dhumale GB, Goyal RC, Phalke DB, Ghodke YD. Depression among elderly persons in a primary health centre area in Ahmednagar, Maharastra. Indian J Public Health 2009;53:253-5.

24. Ganguli M, Ratcliff G, Chandra V, et al. A Hindi version of the MMSE: the development of a cognitive screening instrument for a largely illiterate rural elderly population in India. Int J Geriatr Psychiatry 1995;10:367-77. Crossref

25. Veerapu N, Praveenkumar BA, Subramaniyan P, Arun G. Functional dependence among elderly people in a rural community of Andhra Pradesh, South India. Int J Community Med Public Health 2016;3:1835-40. Crossref

26. Rockwood K, Andrew M, Mitnitski A. A comparison of two approaches to measuring frailty in elderly people. J Gerontol A Biol Sci Med Sci 2007;62:738-43. Crossref

27. Mitnitski AB, Graham JE, Mogilner AJ, Rockwood K. Frailty, fitness and late-life mortality in relation to chronological and biological age. BMC Geriatr 2002;2:1. Crossref 